\title{
Relationship Between Dental Caries and Erosive Tooth Wear in Adolescents
}

\author{
Megan L. Weber ${ }^{1}$, Jenny Bogstad Søvik ${ }^{2}$, Aida Mulic ${ }^{2}$, Kathleen Deeley ${ }^{1}$, \\ Adriana Modesto ${ }^{3}$, Anne B. Tveit ${ }^{2}$ and Alexandre R. Vieira ${ }^{1 *}$ \\ ${ }^{1}$ Department of Oral and Craniofacial Sciences, School of Dental Medicine, University of Pittsburgh, Pittsburgh, PA, \\ United States, ${ }^{2}$ Department of Cariology, Institute of Clinical Dentistry, University of Oslo, Oslo, Norway, ${ }^{3}$ Department of \\ Pediatric Dentistry, School of Dental Medicine, University of Pittsburgh, Pittsburgh, PA, United States
}

\section{OPEN ACCESS}

Edited by:

Raffaella Docimo,

University of Rome Tor Vergata, Italy

Reviewed by:

Jan Kühnisch,

LMU Munich University

Hospital, Germany

Mohammed El-Awady Grawish,

Mansoura University, Egypt

Thais Manzano Parisotto,

Sao Francisco University, Brazi

${ }^{*}$ Correspondence:

Alexandre R. Vieira arv11@pitt.edu

Specialty section:

This article was submitted to

Pediatric Dentistry,

a section of the journal

Frontiers in Dental Medicine

Received: 08 July 2021 Accepted: 30 December 2021

Published: 28 January 2022

Citation:

Weber ML, Søvik JB, Mulic A

Deeley K, Modesto A, Tveit $A B$ and Vieira AR (2022) Relationship Between Dental Caries and Erosive Tooth Wear in Adolescents.

Front. Dent. Med. 2:738443. doi: 10.3389/fdmed.2021.738443
Background: Our aim was to investigate the relationship between caries experience and erosive tooth wear of adolescents.

Methods: We compared the Decayed, Missing, and Filled Teeth (dmft/DMFT) data of 795 adolescents to their erosive tooth wear scores using diet as a covariate and determined if dental caries and erosive tooth wear scores are associated using linear regression analysis. Diet data and oral hygiene habits were collected using selfreported surveys and erosive tooth wear scores were previously collected. We also compared patients' genotypes and phenotypes and looked for an association between erosive tooth wear experience and different single nucleotide polymorphisms (SNPs). A Bonferroni correction was implemented to correct multiple comparisons. Two-group comparisons were made depending on the phenotype definitions implemented, and both chi-square and linear regression analyses were used to test association between genetic variants and caries definitions. All covariates were included in each model.

Results: For four SNPs (rs17159702, rs10246939, rs1800972, and rs1676303), there was an association between a spike in caries experience of DMFT 4 or more between two time points and increased frequency of fruit juice intake. A fifth SNP rs2860216 was shown to be a protective factor toward a spike in caries when coupled with more frequently eating yogurt. We did not find significant associations between our dental caries phenotypes or our demographic data and erosive tooth wear status in our linear regression.

Conclusions: Dental caries and erosive tooth wear are two diseases that differ in mechanism and heritability.

Keywords: dental caries, dental erosion, genetics, adolescent and youth, association studies in genetics

\section{INTRODUCTION}

Erosive tooth wear is caused by acid from food and drink, stomach acid due to vomiting or reflux, occupational exposure, or is idiopathic (1). Soft drinks, juice, and fruits are culprits of erosive tooth wear, especially in younger patients (1-6). Additionally, a genome-wide association study (GWAS) had suggested a genetic influence in the condition (7). An association between enamel formation genes and erosive tooth wear was reported previously $(8,9)$. 
In our previous work, we found that eating sour sweets and drinking acidic beverages (specifically sports drinks) are associated with dental erosive wear in the population, while tooth brushing frequency was not (5). This study was followed by the demonstration that amelogenin and enamelin were associated with having erosive tooth wear (8). Since it was suggested that some genes involved in enamel formation were associated with dental caries and also associated with erosive tooth wear, here we expanded this inquiry to additional genes (8-10). Our hypothesis is that dental caries and erosive tooth wear may be modulated by similar genes. The genetic variants elected to be studied here were shown to be linked to dental caries.

In this report, we tested if associations can be detected when dental caries phenotypes based on longitudinal data (10) were associated with having erosive tooth wear and certain dietary habits.

\section{MATERIALS AND METHODS}

Of 846 adolescents from western Norway (aged 16-18 years) scheduled for dental recall examinations, 795 (94\%) accepted to participate $(10,11)$. This project was approved by the Regionale Komiteer for Medisink og Helsefaglig Forskningsetikk-REK (approval number 2011/1602) and the University of Pittsburgh Institutional Review Board (IRB) (approval number 12110620). Written informed consent was obtained from all participants and/or their parents. These patients were broken down into groups based on longitudinal analysis of their the Decayed, Missing, and Filled Teeth (DMFT/dmft) scores and genotyped to determine if there are certain host genetic factors that associate with dental caries experience. Patients were unrelated, healthy, and had regular access to dental care. Caries experience $\left(\mathrm{D}_{3-5} \mathrm{MFT} / \mathrm{d}_{3-5} \mathrm{mft}\right)$ data were obtained from patient's dental records and collected as part of a routine dental examination. Concerning approximal lesions were recorded during the radiographic examination, only dentin lesions were included $\left(\mathrm{D} / \mathrm{d}_{3-5}\right)$. DMFT/dmft scores were recorded at different ages and depending on the birth year of participants, they had four, five, or six DMFT scores overtime. Dentists treating these children are calibrated by the same professional but no intra-examiner reliability scores were obtained.

These groups created based on the patient's DMFT/dmft can be found in Table 1.

Each DMFT/dmft was collected at a different time point depending on the patient's birth year. DMFT/dmft scores were recorded at different ages and depending on the birth year of participants, they had 4, 5, or 6 DMFT scores overtime. Children born in 1994 had DMFT/dmft data recorded at ages 5, 12, 14, 16, 17, and 18. Children born in 1995 had DMFT/dmft data recorded at ages 5,12,14,16, and 17. Finally, children born in 1996 had DMFT/dmft data recorded at ages 5, 12, 14, and 16. Individuals with low caries experience included subjects with a DMFT of one, two, or three, and individuals with a high caries experience, which included subjects with a DMFT of four or more. We chose our high caries DMFT/dmft scores as four or more due to the mean DMFT/dmft score for the population being
TABLE 1 | Groups created in the previous study based on dmft/DMFT scores.

\begin{tabular}{lc}
\hline Group & Sample size, $\boldsymbol{n}$ \\
\hline No caries (DMFT $=$ 0) vs. low caries (DMFT $\leq$ 3) & 201 vs. 301 \\
No caries vs. high caries (DMFT $\geq 4)$ & 201 vs. 373 \\
Low caries vs. high caries & 301 vs. 373 \\
No primary caries (dmft $=$ 0) vs. low primary caries (dmft $\leq 3)$ & 647 vs. 125 \\
No primary caries (dmft) vs. high primary caries (dmft $\geq 4)$ & 647 vs. 103 \\
Low primary caries (dmft) vs. high primary caries (dmft) & 125 vs. 103 \\
No caries vs. very high caries (DMFT $\geq 8)$ & 201 vs. 128 \\
Very high caries vs. low caries & 301 vs. 128 \\
Very high caries vs. high caries & 245 vs. 128 \\
Acute increase in DMFT (DMFT increased by 4 or more & 128 vs. 527 \\
between time points) vs. no acute increase in DMFT & \\
Acute increase in DMFT vs. no caries & 128 vs. 201 \\
\hline
\end{tabular}

3.5. Patients from the high caries experience group were split into a high caries group with a DMFT of four or more and a very high caries group with a DMFT of eight or more. Eight was chosen as the DMFT cutoff since it is double the number used for high caries experience. We also separated patients based on whether or not they had an acute increase in caries experience between two time points. We called these acute increases "spikes" for short and looked to see if patients experienced more than one over time. In total, 128 patients had a spike of four or more between two time points while the next most common spike was five and occurred in only 42 patients. Therefore, a spike was chosen as an increase in DMFT of four or more between two time points based on our previous determination of high caries experience and based on the distribution of increases between time points across the population.

In this study, the same patient group genotyping results found in previous studies were compared to additional variables, such as diet and oral hygiene behaviors and erosive tooth wear scores. Patients were given self-administered questionnaires to completely describe their oral hygiene and dietary habits at the time the last DMFT score was obtained (5). Prior to initiating the study, the questionnaire was completed by a pilot group $(n=10)$ to ensure comprehension and legibility (5). The survey included questions about brushing, beverage and food consumption, workout habits, and acid regurgitation frequency. A complete list of questions and possible responses are part of the Supplemental Materials.

Some questions had a low frequency of differing responses and were not included in the following analysis. Those included are listed in Table 2. Responses were given scores to be included in the analysis and are also listed in Table 2.

In recall examinations, dental erosive wear for 795 patients of the Norwegian cohort was determined using the Visual Dental erosion Dental Examination scoring system $(5,12)$. Eight calibrated dentists and hygienists collected erosive tooth wear data. Calibrations were performed by the use of photographs and coaching by the same professional. Twenty surfaces of 14 teeth were selected as index surfaces: the occlusal surfaces of the upper and lower first and second molars and the labial 
and palatal surfaces of the upper incisors and canines. Patients with $\geq 3$ index surfaces with dental erosive wear were defined as affected (5). Additional descriptive information on calibration and collection of erosive tooth wear data can be found in previous work $(8,11,12)$. Intraexaminer agreement (kappa) was 0.77, with initial loss of enamel looking to be the most challenging to be scored.

Genotyping was obtained using TaqMan chemistry (13) (Applied Biosystems genotyping probes and master mix, Waltham, MA, USA) and end-point analysis in a 7900HT Fast Real-Time PCR system with 384-well block module (Applied Biosystems, Waltham, MA, USA). Saliva samples were obtained

TABLE 2 | Covariates included in each linear regression test.

\begin{tabular}{|c|c|}
\hline Covariate (questions from survey) & Responses and scoring for analysis \\
\hline Sex & Male $=1$, Female $=2$ \\
\hline Last dental visit & $\begin{array}{l}0-6 \text { months }=1,7-12 \text { months }=2,13-24 \\
\text { months }=3, \text { more than } 24 \text { months }=4\end{array}$ \\
\hline How often patient brushes teeth & $\begin{array}{l}\text { "Rarely" }=1 \text {, "Once daily" = } 2 \text {, "Twice } \\
\text { daily" = 3, "More than twice daily" = } 4\end{array}$ \\
\hline How long patient brushes teeth & $\begin{array}{l}\text { "Less than } 2 \text { min" = 1, "Between } 2 \text { and } 5 \\
\operatorname{min"}=2\end{array}$ \\
\hline $\begin{array}{l}\text { Is a supplemental fluoride source } \\
\text { used? }\end{array}$ & $\begin{array}{l}\text { "Rarely or never" = } 1 \text { "Once weekly" = } 2 \\
\text { "2-3 times per week" = 3, "Daily" = } 4\end{array}$ \\
\hline Frequency of juice intake & $\begin{array}{l}\text { "Rarely or less than one time per week" }= \\
1, " 1-2 \text { times per week" }=2, " 3-5 \text { times } \\
\text { per week" }=3 \text {, "once daily" }=4 \text {, and } \\
\text { "more than once daily" }=5 \text {. }\end{array}$ \\
\hline
\end{tabular}

Frequency of soda/diet soda intake

Frequency of sports drink intake

Frequency of eating citrus fruits

Frequency of eating apples

Frequency of eating chips with dip

Frequency of eating yogurt

Frequency of eating acidic sweets

Erosion (3 or more affected surfaces) $\quad$ Affected $=1$, Unaffected $=2$ using DNA Genotek's saliva-based collection kits (Ottawa, ON, Canada), and DNA was extracted weeks later at the time of genotyping according to the protocol of the manufacturer.

A linear regression was performed using the PLINK software (14) to look for an association between chosen habits which is listed in Table 2 and erosive tooth wear, single nucleotide polymorphisms (SNPs) that were genotyped, and our newly created dental caries phenotypes from a previous study are listed in Table 1 (10).

Additionally, the PLINK software was used to compare patients' genotypes and phenotypes and look for an association between erosive tooth wear experience and the different SNPs from our previous study (10). A Bonferroni correction was implemented to correct for multiple comparisons. Two-group comparisons were made depending on the phenotype definitions implemented, and both chi-square and linear regression analyses were used to test for association between genetic variants and caries definitions. All covariates were included in each model. A flowchart of the steps executed in the methodology is presented in Figure 1.

\section{RESULTS}

A number of SNPs were found to show an association with the covariates as compared with dental caries experience. These are listed in Table 3. Due to the number of $p$ below 0.05 , this table includes only nominal results with $p<0.01$ and the results that hold significant after a Bonferroni correction where the association was set at $p=0.001(0.05 / 49)$. The results significant after Bonferroni are in bold.

No significant associations were found between the erosive tooth wear phenotype and SNPs from genotyping studies.

\section{DISCUSSION}

This study aimed to demonstrate that similar biological factors of the host could be implicated in dental caries and erosive

Recruitment of subjects, clinical evaluations, questionnaires of risk factors and collection of saliva sample

Longitudinal dental caries and erosive tooth wear data obtained from records

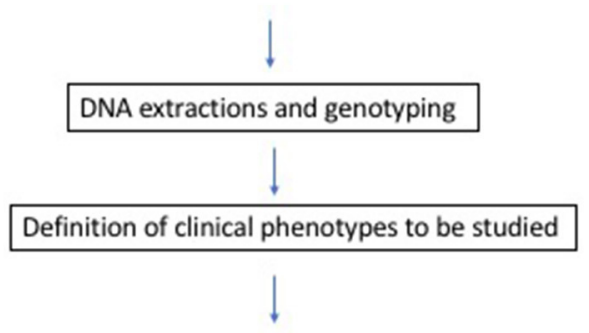

Statistical analyses

FIGURE 1 | Summary of study design. 
TABLE 3 | Summary of the association analysis.

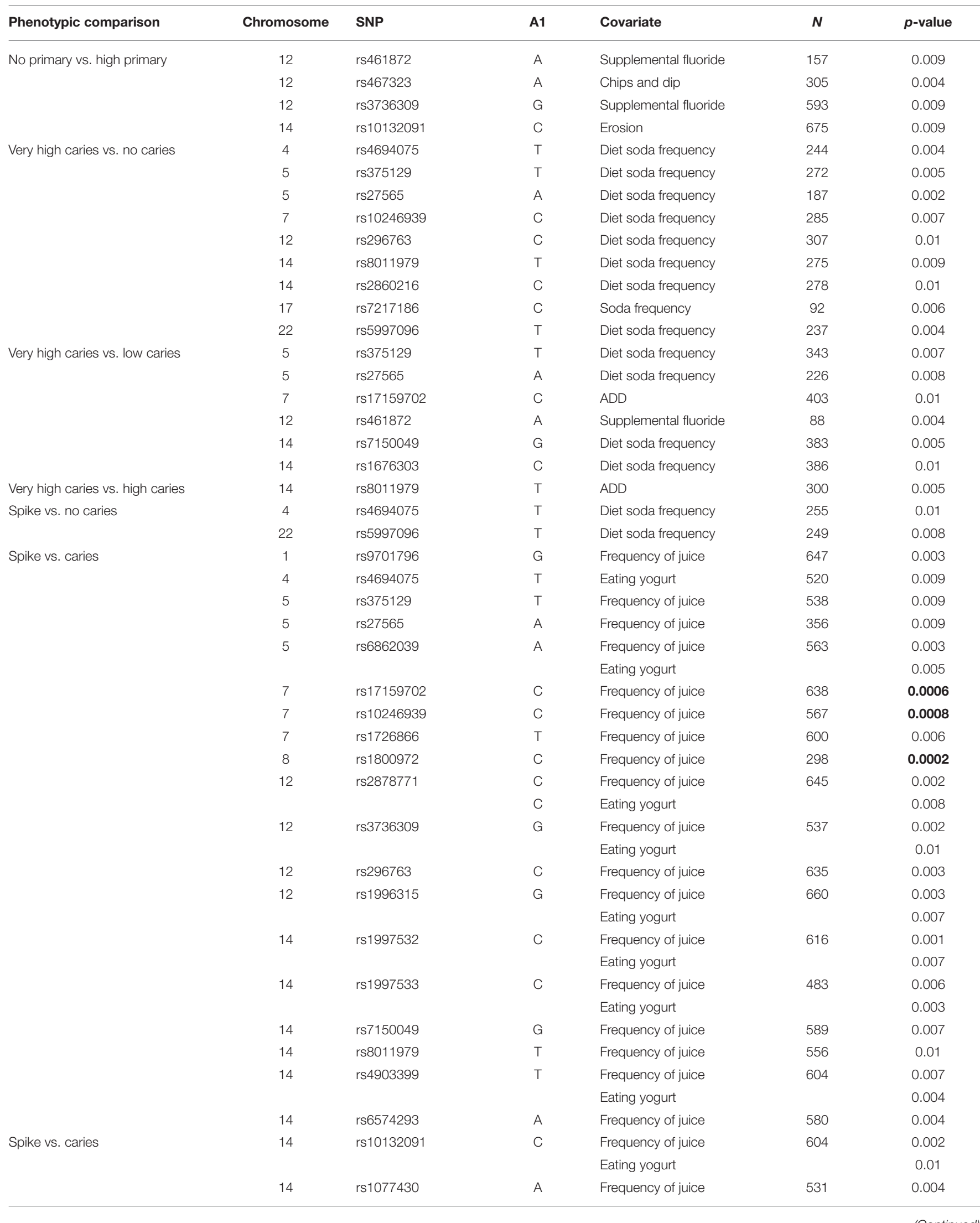


TABLE 3 | Continued

\begin{tabular}{|c|c|c|c|c|c|c|}
\hline Phenotypic comparison & Chromosome & SNP & A1 & Covariate & $N$ & $p$-value \\
\hline & 14 & rs745011 & $\mathrm{C}$ & Frequency of juice & 581 & 0.003 \\
\hline & & & & Eating yogurt & & 0.008 \\
\hline & 14 & rs1676303 & C & Frequency of juice & 615 & 0.0005 \\
\hline & & & & Eating yogurt & & 0.002 \\
\hline & 14 & rs2860216 & C & Frequency of juice & 568 & 0.005 \\
\hline & 19 & rs2235091 & C & Frequency of juice & 613 & 0.002 \\
\hline & & & & Eating yogurt & & 0.002 \\
\hline & 19 & rs198968 & A & Frequency of juice & 550 & 0.006 \\
\hline \multirow[t]{2}{*}{ Spike vs. caries } & 14 & rs10132091 & C & Frequency of juice & 604 & 0.002 \\
\hline & & & & Eating yogurt & & 0.01 \\
\hline
\end{tabular}

Bold indicates Bonferroni corrected significant values.

tooth wear. We attempted to carry out a careful clinical characterization to utilize novel phenotypes for the study of dental caries and erosive tooth wear. After a Bonferroni correction, five results stood out as significant. All of these results were in the comparison of the groups "acute increase vs. caries." In our previous study, we had separated patients based on whether or not they had an acute increase in caries experience (between any two time points) of DMFT of four or more (10). For four SNPs (rs17159702, rs10246939, rs1800972, and rs1676303), there was an association between a spike in caries experience of DMFT 4 or more between two time points and increased frequency of fruit juice intake. SNP rs17159702 is found in aquaporin $1(A Q P 1)$ and therefore may have a role in salivary buffering and dental caries (15). SNP rs10246939 is found in taste 2 receptor member 38 (TAS2R38), which may indicate an association between taste preferences and juice intake (16), SNP rs1800972 is found in defensin beta 1 (DEFB1), an inflammatory response gene $(17,18)$. Finally, SNP rs1676303 is found to flank estrogen-related receptor beta (ESRRB), which may have a developmental role in a patient's dentition (19).

Intake of juices is reported as a major covariate for dental caries and erosive tooth wear occurrence. Studies have shown previously that increased juice intake leads to increased early childhood caries $(20,21)$. Other studies have contradicted this, finding that increased consumption of fruit juice leads to lower instances of dental caries in children (20). However, studies like this one speculate that children who drink less juice may have more dental caries because they are substituting juice with more cariogenic drinks (19). Not as much data are available for adults and the frequency of dental caries in those who drink juice as compared to those who do not. To our knowledge, this is the first study that has looked at the impact of drinking more juice on a subset of patients with an acute increase in dental caries experience between two time points. Therefore, while other studies have not necessarily shown an association between increased juice intake and increased caries experience, these studies have only looked at overall caries experience and not longitudinal data.

Dairy intake is also suggested as a risk modulator of dental caries and erosive tooth wear. A fifth SNP rs2860216 was shown to be a protective factor toward a spike in caries when coupled with more frequent eating yogurt. This SNP lies in ESRRB and has also been shown to play a potential role in caries experience as a gene involved in dental development (19). In previous studies, eating yogurt has been found to potentially hamper the effects of Streptococcus mutans due to its probiotic nature and lower the risk for dental caries (22-24). Again, to our knowledge, this is the first study to show the potential protective role of yogurt consumption in patients that may otherwise experience a spike in dental caries experience.

Another modulator of risks is soda intake. Very high caries experience (DMFT $\geq 8$ ) vs. no caries seems to be borderline associated with increased diet soda intake. This seems counterintuitive since diet soda usually has sugar removed. Studies have agreed that soda causes demineralization of tooth structure and that artificially sweetened soda intake yields less dental caries than full-sugar soda. However, studies have shown that artificially sweetened sodas still have some cariogenic potential (25). Patients may also be drinking more artificially sweetened sodas because they believe they are healthier than regular sodas. This may account for the extra high caries experience in some patients compared to those with no caries.

Diets high in sugar and carbohydrates have been known to play a role in dental caries experience. The Vipeholm studies were some of the earliest studies demonstrating this (26). While erosive tooth wear and dental caries differ in their causation, they are both mediated by diet and therefore the dietary survey responses can be applied to dental caries experience as well (1). We were limited in our dietary analysis because we did not have longitudinal diet data. Data were collected at one point 
in time when the patients were either 16,17 , or 18 years of age. Therefore, it is a bit of a stretch to analyze our primary caries groups with the diet data since it was collected with strictly permanent dentition in the mouth. However, because the patients still live at home and are relatively healthy in their habits, the diets may still reflect those that impacted the primary dentition. Moreover, there are gaps in time between when the caries data were collected and when the diet data were collected. However, by stratifying our patients into groups based on their trends in caries experience rather than caries experience at certain time points, we believe the diet data are still relevant.

However, there were several limitations of this study. Because the questionnaires were self-administered, the results may be affected by the patient's recall bias or selective reporting. Additionally, while the DMFT/dmft data were longitudinal, the diet data were collected at one time point between the ages of 1618. Therefore, there is no information on past dietary preferences or tooth brushing habits (5). However, there was a $94 \%$ response rate for patients across the entire questionnaire so the likelihood of non-response bias is low (5).

For future work, as research of the genetic influences in erosive tooth wear is limited, a GWAS could be indicated as well for that disease. Also, there is additional diet data that we did not analyze in our linear regression. Specifically, the methods of drinking fluids (from glass, bottle, sports bottle, or straw) were not analyzed in this study as they were beyond the scope. They were, however, analyzed in the previous studies of erosive tooth wear (5), where consumption of diet soft drinks from a glass or straw resulted in a significantly lower prevalence of dental erosive wear compared to drinking from a bottle. For the other acidic beverages, no significant associations were found when comparing the chosen drinking method and erosive tooth wear. It may be an interesting pursuit to determine if methods of drinking acidic or cariogenic beverages (i.e., through a straw, and swishing in the mouth) affect dental caries experience. Additionally, our study concludes that dental caries and erosive tooth wear are two diseases that differ in mechanism and heritability. This study may provide insight toward the

\section{REFERENCES}

1. Fejerskov O, Kidd E. Dental Caries: The Disease And Its Clinical Management. Oxford: John Wiley \& Sons (2008).

2. Jensdottir I, Holbrook P, Nauntofte B, Buchwald C, Bardow A. Immediate erosive potential of cola drinks and orange juices. J Dent Res. (2006) 85:22630. doi: $10.1177 / 154405910608500304$

3. Cheng $\mathrm{R}$, Yang $\mathrm{H}$, Shao $\mathrm{M}, \mathrm{Hu} \mathrm{T}$, Zhou $\mathrm{X}$. Dental erosion and severe tooth decay related to soft drinks: a case report and literature review. J Zhejiang Univ Sci B. (2009) 10:395-9. doi: 10.1631/jzus.B082 0245

4. Mulic A, Skudutyte-Rysstad R, Tveit AB, Skaare AB. Risk indicators for dental erosive wear among 18-yr-old subjects in Oslo, Norway. Eur J Oral Sci. (2012) 120:531-8. doi: 10.1111/j.1600-0722.2012.00997.x

5. Sovik J, Skudutyte-Rysstad R, Tveit AB, Sandvik L, Mulic A. Sour sweets and acidic beverage consumption are risk indicators for dental erosion. Caries Res. (2015) 49:243-50. doi: 10.1159/000371896 heritability of erosive tooth wear and that despite both being diseases that stem from demineralization of the hard tissues of teeth, they are in fact different from one another.

\section{DATA AVAILABILITY STATEMENT}

The original contributions presented in the study are included in the article/Supplementary Materials, further inquiries can be directed to the corresponding author.

\section{ETHICS STATEMENT}

The studies involving human participants were reviewed and approved by University of Pittsburgh Institutional Review Board. Written informed consent to participate in this study was provided by the participants' legal guardian/next of kin.

\section{AUTHOR CONTRIBUTIONS}

MW and AV conceived and planned the experiments. MW carried out the experiments and took the lead in writing the manuscript. JS, $\mathrm{AMu}, \mathrm{KD}$, and $\mathrm{AT}$ contributed to sample preparation. MW, AMo, and AV contributed to the interpretation of the results. All authors provided critical feedback and helped to shape the research, analysis, and manuscript. These data is part of a thesis of MW submitted as part of the requirements to fulfilment of a Ph.D. in Oral Biology.

\section{FUNDING}

Funding for this study provide from NIH/NIDCR R01-DE18914, the University of Oslo, and graduate support from the University of Pittsburgh, School of Dental Medicine.

\section{SUPPLEMENTARY MATERIAL}

The Supplementary Material for this article can be found online at: https://www.frontiersin.org/articles/10.3389/fdmed. 2021.738443/full\#supplementary-material

6. Richards D. Impact of diet on tooth erosion. Evid Based Dent. (2016) 17:40. doi: 10.1038/sj.ebd.6401164

7. Sovik J, Vieira AR, Tveit AB, Mulic A. Enamel formation genes associated with dental erosive wear. Caries Res. (2015) 49:236-42. doi: 10.1159/000369565

8. Uhlen MM, Mulic A, Holme B, Tveit AB, Stenhagen KR. The susceptibility to dental erosion differs among individuals. Caries Res. (2016) 50:11723. doi: $10.1159 / 000444400$

9. Alaraudanjoki VK, Koivisto S, Pesonen P, Männikkö M, Leinonen J, Tjäderhane L, et al. Genome-wide association study of erosive tooth wear in a Finish cohort. Caries Res. (2019) 53:49-59. doi: 10.1159/000488208

10. Weber M, Bogstad Søvik J, Mulic A, Deeley K, Tveit AB, Forella J, et al. Redefining the phenotype of dental caries. Caries Res. (2018) 52:26371. doi: $10.1159 / 000481414$

11. Sovik J, Tveit AB, Storesund T, Mulic A. Dental erosion: a widespread condition nowadays? A cross-sectional study among a group of adolescents in Norway. Acta Odontol Scand. (2014) 72:523-9. doi: 10.3109/00016357.2013.875588 
12. Mulic A, Tveit AB, Wang NJ, Hove LH, Espelid I, Skaare AB. Reliability of two clinical scoring systems for dental erosive wear. Caries Res. (2010) 44:294-9. doi: 10.1159/000314811

13. Ranade K, Chang MS, Ting CT, Pei D, Hsiao CF, Olivier M, et al. Highthroughput genotyping with single nucleotide polymorphisms. Genome Res. (2001) 11:1262-8. doi: 10.1101/gr.157801

14. Purcell S, Neale B, Todd-Brown K, Thomas L, Ferreira MAR, Bender D, et al. PLINK: a tool set for whole-genome association and population-based linkage analyses. Am J Hum Genet. (2007) 81:559-75. doi: 10.1086/519795

15. Bezamat M, Cunha RJ, Modesto A, Vieira AR, Taboas JM. Aquaporin locus (12q13.12) might contribute to susceptibility of temporomandibular joint associated with periodontitis. PLOS ONE. (2020) 15:e0229245. doi: 10.1371/journal.pone.0229245

16. Wendell S, Wang X, Brown M, Cooper ME, DeSensi RS, Weyant RJ, et al. Taste genes associated with dental caries. J Dent Res. (2010) 89:1198202. doi: $10.1177 / 0022034510381502$

17. Ozturk A, Famili P, Vieira AR. The antimicrobial peptide DEFB1 is associated with caries. J Dent Res. (2010) 89:631-6. doi: 10.1177/002203451036 4491

18. Krasone K, Lace B, Akota I, Care R, Deeley K, Küchler EC, et al. Genetic variation in the promoter region of beta-defensin 1 (DEFB 1) is associated with high caries experience in children born with cleft lip and palate. Acta Odontol Scand. (2014) 72:235-40. doi: 10.3109/00016357.2013.8 22549

19. Weber ML, Hsin H-Y, Kalay E, Brozkova DS, Shimizu T, Bayram M, et al. Role of estrogen related receptor beta (ESRRB) in DFN35B hearing impairment and dental decay. BMC Med Genet. (2014) 15:81. doi: 10.1186/1471-2350-15-81

20. AbdelAziz WE, Dowidar KML, El Tantawi MMA. Association of healthy eating, juice consumption, and bacterial counts with early childhood caries. Pediatr Dent. (2015) 37:462-7.

21. Catteau C, Trentesaux T, Delfosse C, Rousset M-M. Consumption of fruit juices and fruit drinks: impact on the health of children and teenagers, the dentist's point of view. Arch Pediatr. (2012) 19:11824. doi: 10.1016/j.arcped.2011.11.011
22. Twetman S, Stecksén-Blicks C. Probiotics and oral health effects in children. Int J Paediatr Dent. (2008) 18:3-10. doi: 10.1111/j.1365-263X.2007.00912.x

23. Varghese L, Varughese JM, Varghese NO. Inhibitory effect of yogurt extract on dental enamel demineralisation - an in vitro study. Oral Health Prev Dent. (2013) 11:369-74.

24. Yadav M, Poornima P, Roshan NM, Prachi N, Veena M, Neena IE. Evaluation of probiotic milk on salivary mutans streptococci count: an in vivo microbiological study. J Clin Pediatr Dent. (2014) 39:236. doi: 10.17796/jcpd.39.1.u433n8w245511781

25. Giacaman RA, Campos P, Munoz-Sandoval C, Castro RJ. Cariogenic potential of commercial sweeteners in an experimental biofilm caries model on enamel. Arch Oral Biol. (2013) 58:1116-22. doi: 10.1016/j.archoralbio.2013.03.005

26. Gustafsson B, Quensel C, Lanke L, Lundqvist C, Grahnen H, Bonow B, et al. The Vipeholm dental caries study; the effect of different levels of carbohydrate intake on caries activity in 436 individuals observed for five years. Acta Odontol Scand. (1954) 11:232-64. doi: 10.3109/00016355308993925

Conflict of Interest: The authors declare that the research was conducted in the absence of any commercial or financial relationships that could be construed as a potential conflict of interest.

Publisher's Note: All claims expressed in this article are solely those of the authors and do not necessarily represent those of their affiliated organizations, or those of the publisher, the editors and the reviewers. Any product that may be evaluated in this article, or claim that may be made by its manufacturer, is not guaranteed or endorsed by the publisher.

Copyright (C) 2022 Weber, Søvik, Mulic, Deeley, Modesto, Tveit and Vieira. This is an open-access article distributed under the terms of the Creative Commons Attribution License (CC BY). The use, distribution or reproduction in other forums is permitted, provided the original author(s) and the copyright owner(s) are credited and that the original publication in this journal is cited, in accordance with accepted academic practice. No use, distribution or reproduction is permitted which does not comply with these terms. 